\title{
BMJ Open Testing the feasibility and acceptability of using the Nintendo Wii in the home to increase activity levels, vitality and well-being in people with multiple sclerosis (Mii-vitaliSe): protocol for a pilot randomised controlled study
}

\section{Sarah Thomas, ${ }^{1}$ Louise Fazakarley, ${ }^{2}$ Peter W Thomas, ${ }^{1}$ Sarah Brenton, ${ }^{3}$ Sarah Collyer, ${ }^{3}$ Steve Perring, ${ }^{4}$ Rebecca Scott, ${ }^{5}$ Kathleen Galvin, ${ }^{6}$ Charles Hillier ${ }^{3}$}

To cite: Thomas $S$,

Fazakarley L, Thomas PW, et al. Testing the feasibility and acceptability of using the Nintendo Wii in the home to increase activity levels, vitality and well-being in people with multiple sclerosis (MiivitaliSe): protocol for a pilot randomised controlled study. BMJ Open 2014:4:e005172. doi:10.1136/bmjopen-2014005172

- Prepublication history and additional material is available. To view please visit the journal (http://dx.doi.org/ 10.1136/bmjopen-2014005172).

Received 3 March 2014 Accepted 27 March 2014

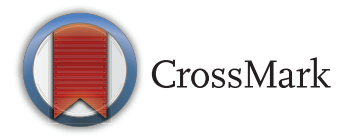

For numbered affiliations see end of article.

Correspondence to Dr Sarah Thomas; saraht@bournemouth.ac.uk

\section{ABSTRACT}

Introduction: The benefits of physical activity for people with multiple sclerosis (pwMS) have been recognised. However, exercise regimens can be difficult to maintain over the longer term and pwMS may face unique barriers to physical activity engagement. Pilot research suggests the Nintendo Wii can be used safely at home by pwMS with minimal mobility/balance issues and may confer benefits. We have developed a home-based physiotherapist supported Wii intervention ('Mii-vitaliSe') for pwMS that uses commercial software. This is a pilot study to explore the feasibility of conducting a full scale clinical and cost-effectiveness trial of Mii-vitalise.

Methods and analysis: 30 ambulatory, relatively inactive pwMS will be randomised to receive Mii-vitaliSe immediately, or after 6 months. Outcomes, measured at baseline and 6 and 12 months later, will include balance, gait, mobility, hand dexterity and self-reported physical activity levels, fatigue, self-efficacy, mood and quality of life. Interviews conducted on a purposive sample of participants will explore experiences of participation in the study and barriers and facilitators to using the Wii. Mean recruitment, adherence rate and standard deviations (SDs) of potential primary outcomes for the full trial will be estimated and precision summarised using $95 \%$ confidence intervals (Cls). Interview transcripts will be thematically analysed using a generic qualitative approach.

Ethics and dissemination: National Health Service (NHS; ref 12/SC/0420) and university ethical approvals have been obtained as has NHS Research and Development permission from the relevant trust. A home risk assessment will be undertaken for all potential participants. All adverse events will be closely monitored, documented and reported to the study Safety Monitoring Committee. At least one publication in a peer reviewed journal will be produced and research findings presented at a national and international conference. With service users, we will coproduce a summary of the findings for dissemination on our research unit's website and elsewhere.

Trial registration number: ISRCTN 49286846.
Strengths and limitations of this study

- This pilot study will include a long-term (1 year) follow-up to consider adherence.

- The intervention will incorporate individual tailoring, behavioural change strategies and homebased Wii use with physiotherapist guidance.

- Mixed methods will be used.

- Physical outcome assessments will not be blinded.

\section{INTRODUCTION}

Historically, people with multiple sclerosis (pwMS) have been advised to limit physical activity levels. However, over the last decade the salutary effects of exercise for pwMS have become evident, ${ }^{1-5}$ with no deleterious effects described. ${ }^{1}{ }^{6-9}$ Physical activity has been shown to be associated with improvements in mobility, muscle strength and physical fitness. ${ }^{19-14}$ In a meta-analysis, planned and structured regular exercise was associated with a small improvement in quality of life (QoL) in MS. ${ }^{15}$ Other secondary benefits might include reduced fatigue, depression and anxiety and improved sense of wellbeing-although more rigorous trials are needed. ${ }^{14}{ }^{16}$ Early intervention may help to slow or prevent the accumulation of physical disability. ${ }^{17} 18$

However, pwMS typically engage in dramatically lower levels of physical activity than the general population. ${ }^{2} 419$ This can lead to a downward spiral of deconditioning, fatigue, reduced self-efficacy, increased levels of disability ${ }^{4}$ as well as increased health risks (such as cardiovascular diseases, obesity, diabetes, etc). Psychosocial repercussions include reduced leisure, social contacts, role 
fulfilment and engagement in activities of daily living; all important for self-esteem and psychological well-being.

People with long-term disabling conditions such as MS face unique barriers to participation in physical activity $^{20-28}$ including physical (eg, pain, fatigue, mobility limitations and overheating), psychological (eg, fear, embarrassment and lack of confidence) and environmental (transport, cost, lack of suitable facilities/trained staff) factors. While home-based activities overcome some of these, adherence to traditional exercise programmes is poor. ${ }^{29}$ Making exercise an enjoyable experience $^{30-32}$ undertaken in a non-threatening and comfortable environment along with the provision of detailed guidance, monitoring and support may improve adherence. $^{2} 303233$

The National Institute for Health and Care Excellence (NICE) guidelines, the National Service Framework for long-term conditions and the Health Foundation highlight the importance of guided tailored selfmanagement supported by health professionals ${ }^{34-38}$ to encourage engagement in physical activity and leisure and social pursuits.

Physical activity is multifaceted and psychological factors play a crucial role in its initiation and maintenance. Adopting a multidisciplinary approach can help to support behaviour change and physical activity prescription. Social Cognitive Theory ${ }^{39}$ and in particular, the concept of self-efficacy ${ }^{40}$ has been proposed as a key mediator in the promotion and maintenance of physical activity $^{42} 43$ and adherence ${ }^{32} 44-46$ and an important predictor of health status. ${ }^{47}$

The Wii overcomes some of the barriers to participation in physical activity highlighted earlier. It is low cost, can be used at home, and has broad appeal, incorporating variables known to enhance motivation, selfefficacy $^{48}$ and adherence (such as self-monitoring, prompts, peer comparison and feedback about progress and performance) in the context of fun ${ }^{49-52}$ purposeful tasks. ${ }^{53}$ It offers opportunities for socialisation across the lifespan $^{54} 55$ and participation in immersive virtual reality activities that might, in real life, be challenging due to physical limitations, or require further adaptation to enable participation (eg, golf). Games such as Wii Sports use significantly more energy than sedentary activities $^{56-60}$ (though not as much as playing the sport itself). ${ }^{57} 58$

For the current study the Wii was deemed preferable over other newer more complex virtual reality platforms, such as the Xbox Kinect: it is low cost, requires less space, permits support from a therapist within the field of view and the Wii sensors are relatively 'forgiving' thereby facilitating use by people with impaired or compromised movement. ${ }^{61}$ Our findings will, nevertheless, have applicability to and relevance for other platforms and chronic conditions.

In conjunction with the Wii balance board, the Wii Fit Plus incorporates games requiring the user to shift his/her weight from side to side and forwards and back to control gameplay. A key feature of these systems is the provision of dynamic visual feedback about centre of gravity allowing training and adjustments of movement via biofeedback techniques. These recent advances in interactive gaming have created the potential for increasing physical activity and decreasing sedentary behaviour. ${ }^{62}$ It has been suggested that such games may be helpful for improving balance and core strength ${ }^{63}$ under the guidance of a qualified health/rehabilitation professional (who can advise on the selection of appropriate activities to challenge balance safely and gradually).

Wii games can be played for brief periods, at the individual's convenience. Daily activity levels can be accumulated in short bouts of $10 \mathrm{~min}$ or more throughout the day (which is thought to be as effective as all in one go $^{43}{ }^{64}$ ), at differing levels, and a range of activities can be selected, depending on current symptoms ${ }^{65}$ and preferences. Creating a personalised representation of oneself (a 'Mii') may enhance self-efficacy via a greater sense of 'presence' and motivation. ${ }^{66}$

However, there are potential disadvantages. As with other physical activities, playing the Wii could lead to injury, discomfort ${ }^{67} 68$ or increased symptoms. Many of the reported injuries have been due to people exercising beyond their limitations and without resting adequately. The user manual ${ }^{69}$ provides guidance on how to use the Wii safely and onscreen prompts about resting are regularly provided. As the Wii software has been developed with a healthy population in mind, it is important to explore its acceptability to pwMS. ${ }^{70}$

While applications of the Wii are diverse ${ }^{71-82}$ and preliminary findings positive, the evidence base is limited and definitive answers require well-designed randomised controlled trials (RCTs). In this pilot study, we wish to explore whether a commercially available interactive gaming system, the Nintendo Wii, offers a safe, feasible way to provide opportunities in the home for increased physical activity for pwMS (which could lead to improvements in domains such as vitality, balance, mobility, QoL and well-being). To date, Wii research with people affected by MS is mostly preliminary. Early findings are promising, however, and suggest that the Wii might be helpful for balance ${ }^{83-85}$ and fitness. ${ }^{86}$

In a non-randomised pilot study Plow and Finlayson, ${ }^{86}$ found that pwMS who have mild balance and mobility limitations can use the Wii Fit safely at home. However, they also reported that Wii use declined after 3 months. They suggested that a more person-centred approach as well as the use of behavioural change techniques (such as motivational interviewing and problem solving) might help to promote longer term Wii use and foster intrinsic motivation. In a scoping review of the exergaming literature Plow $e t a l^{87}$ recommended that trials incorporate longer term follow-up periods, employ mixed methods and follow patients from the clinical setting to the home. Others have suggested that an individualised approach is essential to take into account individuals' 
beliefs, self-efficacy and perceptions about exercise ${ }^{31}$ and their self-identity, goals and expectations for improving health. ${ }^{70} 88$

Often research projects proceed rapidly to a full scale RCT, with vital, preparatory phases overlooked. ${ }^{89}$ This can result in a resource intensive and costly analysis of a conceptually or clinically flawed intervention. In this study, we have consulted with pwMS already using the Wii, and adapted existing guidance on using the Wii to develop a home-based physiotherapist supported Wii intervention for pwMS (Mii-vitaliSe). We will undertake a small pilot RCT of the Mii-vitaliSe intervention to explore the feasibility of a full-scale formal evaluation of effectiveness. ${ }^{90}$

\section{METHODS}

\section{Aim}

To investigate the feasibility of conducting a multicentre definitive RCT to assess the clinical and costeffectiveness of a home-based physiotherapist supported Wii intervention (Mii-vitaliSe) for pwMS.

\section{Objectives}

1. Test procedures (including administration of the selfreported outcomes and the physical assessments, delivery of the intervention, recording and monitoring of adverse events (AEs)), estimate recruitment and retention rates, and refine the selection of outcome measures in preparation for a definitive RCT to test the effectiveness of Mii-vitaliSe.

2. Collect data on the variability of outcome measures to inform a sample size calculation for a larger trial.

3. Estimate adherence rates via a self-reported daily play $\log$ (in terms of frequency, intensity, duration).

4. Determine the acceptability of randomisation and of the Mii-vitaliSe intervention to participants and obtain information about patterns of use/barriers to use.

5. Gather feedback from physiotherapists about their experiences of delivering Mii-vitaliSe.

\section{Study design}

A single centre wait-list controlled pilot study of Mii-vitaliSe plus usual care versus usual care alone (see figure 1) will be undertaken. Participants will be randomised to receive Mii-vitaliSe immediately or after 6 months ('delayed' group). The delayed group will continue their usual routines and receive the Mii-vitaliSe intervention after a 6-month delay. During this period, they will be asked to refrain from using the Wii and will act as controls for the immediate group.

This design has the potential to minimise the impact of disappointment that those allocated to a nonintervention group might experience, while ensuring those in the delayed group receive the full intervention with an emphasis on safety aspects. Delivering the intervention to those in the delayed group may increase

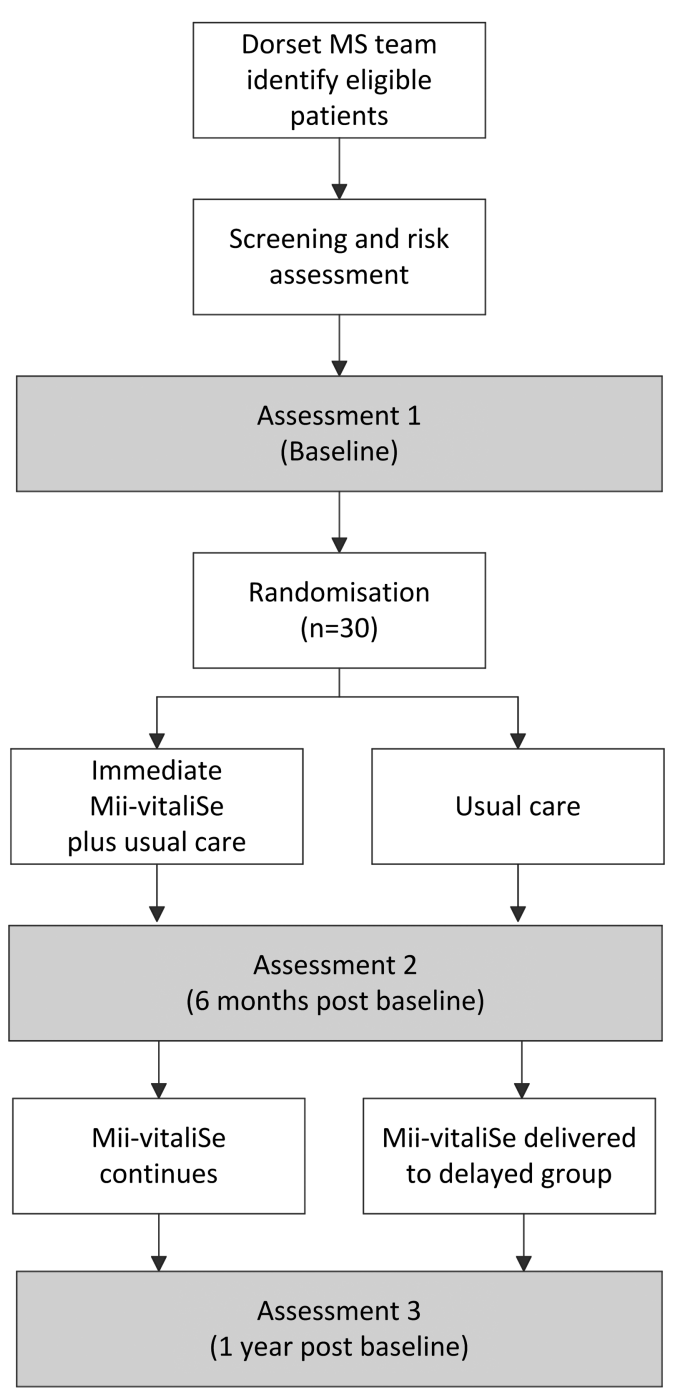

Figure 1 Mii-vitaliSe study flow chart.

compliance with refraining from use for a 6-month period. This design also permits a preliminary consideration of long-term (1 year) follow-up (immediate group only). The randomised controlled component allows testing of procedures, design, etc in preparation for a full scale trial. Follow-up periods are sufficient for novelty value to diminish and patterns of use to be established.

\section{Service user involvement}

Following INVOLVE principles, ${ }^{91}$ services users have been involved in all stages of the research so far, including the development of this protocol. A service user consultation was held to inform the content and format of the Mii-vitaliSe materials and aspects of the study design. Service users will be represented on the trial steering group and will continue to provide ongoing input into the study.

\section{Data protection and data storage}

All information collected during the course of the study will be kept strictly confidential and any information that leaves Poole Hospital/Bournemouth University will 
contain no personal details. Questionnaires will be allocated a participant ID; they will not contain names or any identifying details. Only authorised members of the research team will have access to the study data.

Data will be collected and retained in accordance with the Data Protection Act 1998. Study documents (paper and electronic) will be retained in a secure location during and after the trial has finished. All source documents will be retained for a period of 5 years following the end of the study.

\section{Compliance}

The Chief Investigator will ensure that the study is conducted in compliance with the principles of the Declaration of Helsinki (1996) and in accordance with all applicable regulatory requirements including, but not limited to, the Research Governance Framework and Trust policies and any subsequent amendments.

\section{Sample size considerations}

A 'rule of thumb' of 30 is common for pilot studies. ${ }^{92} \mathrm{~A}$ sample size of 30 (15 in the immediate and 15 in the delayed arm) will be adequate given the specific quantitative aims of this pilot.

Objective 1 is to estimate the recruitment rate to help in planning recruitment for a full scale RCT. Precision of this estimate will be based on the width of the $95 \%$ CI. With a total sample size of 30 people recruited, the recruitment rate will be estimated with a precision of $\pm 11 \%$ (assuming a recruitment rate of approximately $40 \%$ based on experiences of running a RCT of a fatigue management programme for pwMS ${ }^{93}$ ).

Objective 2 is to estimate the SD of potential primary outcome measures in preparation for a formal sample size calculation for a larger RCT. Assuming these outcomes are standardised, so that they have a SD of 1, the precision (as summarised by standard error) will be 0.13 . When using this estimate for a later sample size calculation it will be inflated as suggested by Browne. ${ }^{94}$ Estimating the effect size for the Mii-vitaliSe intervention is not an aim of this pilot, but would be the aim of a later full scale RCT.

Objective 3 is to estimate the degree of adherence of participants to Mii-vitaliSe. For each individual this will be summarised as the number of days on which activities were performed divided by the number of days agreed with the physiotherapist in their action plan. The potential range is $0-1$, and the SD will be at most 0.17 (NQuery Advisor). With a sample size of 30, average adherence will be estimated with precision \pm 0.06 (ie, width of $95 \% \mathrm{CI}$ ).

\section{Participants}

Thirty 'inactive' (see eligibility criteria) pwMS aged 18 or over, will be recruited from the Dorset MS Service. Individuals excluded from the research project will continue to be seen as per usual care.
Eligibility criteria

Inclusion criteria

- Clinically definite diagnosis of MS

- Aged 18 or above

- Fulfil home risk assessment criteria (see below)

- 'Inactive' (physically active on fewer than 5 days/ week for $\geq 30 \mathrm{~min}$ )

- Living within Poole/Bournemouth conurbations

- Suitable television

\section{Exclusion criteria}

- Have only mild symptoms (equivalent to an Adapted Patient Determined Disease Steps (APDDS) Scale ${ }^{95}$ score of 1) or require at least intermittent or unilateral constant assistance (cane, crutch or brace) to walk $100 \mathrm{~m}$ with or without resting (equivalent to an Expanded Disability Status Scale (EDSS) score $^{96}$ of 6 or more)

- Relapse within the past 3 months requiring corticosteroids

- Already participating in exercise/rehabilitation research

- Any medical condition placing participant at risk from exercise participation/using Wii

- Owns a Wii and uses it regularly (weekly or more)

- Unwilling/unable to comply with protocol

\section{Risk assessment}

This will be undertaken at home by the study researcher (who is a senior physiotherapist) to assess balance on the Wii balance board and the suitability of the home environment for setting up the equipment and using the Wii.

Participants will be considered eligible if:

- They can maintain independent standing balance with eyes open for $1 \mathrm{~min}$.

- The physiotherapist clinically judges that they demonstrate adequate balance reactions while on the Wii balance board ${ }^{97}$ and are able to step off safely forwards, backwards and sideways.

- Their home environment is suitable (with minor modifications, if appropriate and possible).

\section{Identification and recruitment}

Recruitment will be via the Dorset MS Service. Potential participants will be identified by the multidisciplinary Dorset MS team. A member of the MS team will go through a screening checklist to identify potential participants and will then either send or give them an invitation letter with a simple one-page summary ('Key Facts') about the study, participant information sheets, the APDDS Scale, a question about current levels of physical activity and a reply slip and pre-paid return envelope. Participants will be asked to return the reply slip and their responses if they wish to find out more about the study. A follow-up reminder letter with another copy of the information pack will be sent if a reply is not received within 2 weeks. 


\section{Screening procedure}

The study researcher will telephone those who have expressed an interest in the research, describe the study in more detail, answer questions and go through the remaining screening criteria (see section 'Exclusion criteria') over the telephone. Those not eligible will be informed over the telephone. Individuals who fulfil the study criteria will be visited at home for the risk and home suitability assessment. Minor modifications to the home environment to facilitate the safe use of the Wii balance board will be discussed with the potential participant and documented.

Individuals not eligible to take part will continue to be seen as per usual care.

\section{Informed consent process}

Informed consent will be taken by the Good Clinical Practice (GCP) accredited study researcher in individuals' homes, providing they satisfy the risk and home assessment criteria.

\section{Randomisation}

To ensure good allocation concealment, random allocation will be email based and administered by the study statistician. Randomisation will be carried out on a 1:1 basis and will utilise a computer-based random sequence generator. Variable-sized blocks will be used to ensure approximately equal numbers in the two trial arms. No stratification will be used.

\section{Outcome measures}

As this is feasibility work, a broad range of outcomes has been included. Participants will be requested to complete the outcomes even if they stop using the Wii. A detailed description of outcome measures is included in the web supplement.

Outcomes will be assessed at baseline and at 6 and 12 months. Physical assessments (administered at similar times of the day to reduce the confounding effect of fatigue ${ }^{98}$ ) will be undertaken at the hospital by the study researcher and a clinical scientist. Self-reported questionnaires (presented in a large font) will be completed by participants in their homes, at their own convenience and pace.

\section{Demographic/descriptor variables}

Age, sex, education, employment, marital status, ethnicity, household composition, time since diagnosis, disease course, relapse history, medication, comorbidities.

\section{Adverse events}

Defined as any undesirable outcome, such as injury, falls, discomfort, pain, relapse. These will be recorded and reviewed by an independent Safety Monitoring Committee (comprising a neurologist and a physiotherapist).

\section{Balance, gait mobility}

- Two-minute walk test ${ }^{99}$

- Step test ${ }^{100}$
- Steady stance test ${ }^{101}$

- Instrumented Timed Up and Go (ITUG) ${ }^{102}$

- Gait stride-time rhythmicity ${ }^{103}$

- Static posturography (Limits of Sway) ${ }^{104}$

Physical activity

- Godin Leisure-Time Exercise Questionnaire (GLTEQ) ${ }^{105106}$

ActivPAL ${ }^{107}$

Hand dexterity/coordination

- Nine-hole peg test ${ }^{108}$

Self-efficacy

- The Spinal Cord Injury Exercise Self Efficacy Scale $(\text { SCI-ESES })^{22} 109$

- The Multiple Sclerosis Self-Efficacy (MSSE) Scale ${ }^{110}$

Psychological well-being and QoL

- Hospital Anxiety and Depression Scale (HADS) ${ }^{111}$

- EuroQual 5 Dimensions-5 Levels (EQ-5D-5L) ${ }^{112}$

- Multiple Sclerosis Impact Scale (MSIS-29) ${ }^{113} 114$

- The Fatigue Symptom Inventory (FSI) ${ }^{115}$

- The Medical Outcomes Short-Form Survey V.2 (SF-36v2) ${ }^{116}$

\section{Adherence}

Measuring adherence is challenging ${ }^{117} 118$ and while some have relied on the data captured by the Wii console, this approach is problematic (eg, other family members could use a participant's Mii). In this study, adherence will be defined as the total number of days on which activities are performed divided by the number of days agreed with the physiotherapist in the action plan. We will also collect information related to duration and intensity ${ }^{119120}$ as well as the data stored on the Wii console.

\section{Process measures}

- Daily play log (date/time, whether played, AEs (eg, pain, tenderness, soreness, fatigue, dizziness, headaches, aching, stiffness, falls), activity, duration, intensity, ${ }^{119} 120$ enjoyment, fatigue, reasons for non-use, free-text comments).

- Physiotherapist feedback: Participant contacts will be recorded using a template. At the end of the intervention period the physiotherapists will be interviewed about the ease of delivering the intervention and any problems encountered.

- Semistructured interviews will be conducted (at home/ via telephone) on a purposive sample of participants selected to represent factors pertinent to expectations/experiences of participating in the study and receiving Mii-vitaliSe (such as age, gender, employment status, disease severity, prior experience of home console systems). These will comprise $50 \%$ of participants from each group at the 6-month follow-up and at the 12-month follow-up, the 'immediate' group only. 


\section{Health utilisation}

Economic evaluation would be an important component of future research. We will pilot an existing measure of healthcare utilisation developed for a RCT of a fatigue management programme. ${ }^{93}$ We will provide participants with a resource use log to aid completion of the questionnaire.

\section{Mii-vitaliSe intervention}

Drawing upon a literature review, existing materials ${ }^{121-125}$ and our knowledge of best practice in physiotherapy and psychological interventions in MS, guidance and resource materials for using the Wii were developed. The research team worked in partnership with a service user panel (8 patients from the Dorset MS Service with experience of using the Wii and familiarity with the Wii Sports and Wii Fit Plus software). A workshop was held to discuss the Mii-vitaliSe intervention. The following aspects were discussed:

1. Safety aspects, patterns of usage, benefits/drawbacks, problems identified and whether/how they could be overcome.

2. Factors contributing to longer term use.

3. Feedback about the Wii Sports and Wii Fit Plus software.

4. Which outcome domains are most relevant/meaningful to service users.

5. Feedback on the format and content of a draft daily play log.

After an iterative process of revision, LF and ST produced a set of materials to be used as part of a physiotherapist-supported home-based Wii intervention (Mii-vitaliSe). These include:

- General information about the Wii and Mii-vitaliSe;

- Safety and technical information;

- Guidance on warm up/cool down exercises;

- A guidance book and personal activity workbook;

- Games descriptors for Wii Sports, Wii Sports Resort and Wii Fit Plus;

- Tips on goal-setting and overcoming barriers;

- Signposts to useful resources (eg, websites, forums);

- Quotations from pwMS who attended the consultation workshop about their experiences of using the Wii.

The Mii-vitaliSe intervention is underpinned by principles of self-management, social cognitive, motivational interviewing and cognitive behavioural theories. It incorporates a workbook to enable individually tailored goal setting, monitoring and the identification of facilitators and barriers to engagement with Mii-vitaliSe.

\section{Usual care}

The Dorset MS service offers pwMS multidisciplinary support. Patients are reviewed annually by the team at an outpatient clinic or home visit appointment. This includes a medical review by a neurologist and specialist MS nurse and a therapy review by a physiotherapist and occupational therapist. On completion of the review and necessary assessments, medical and therapy treatment is

\section{Mii-vitaliSe intervention}

Session 1: Orientation to the Wii in the hospital setting (week 1) The physiotherapist goes through the guidance book with the participant to cover:

- The benefits of physical activity

- A practical demonstration of using the software (Wii Sports, Wii Sports Resort and Wii Fit Plus) safely

- Creating a 'Mii'

- Familiarisation with the controls

Session 2: Training and individualised assessment in the hospital setting (week 2)

Participants will be guided through warm up/cool down exercises and given the opportunity to try out activities safely under supervision. The physiotherapist and participant will discuss appropriate activities, taking into account physical capabilities, and personal preferences.

Session 3: Installation of Wii at home and starting individual programme (week 3)

The physiotherapist will install the Wii equipment in participants' homes to interface with their television. Participants will be provided with Wii Sports, Wii Sports Resort and Wii Fit Plus, asked to use their Mii when playing, and to log any other software used via the daily play log. The physiotherapist will reiterate safety advice (eg, the importance of the safety strap, taking a graded approach and frequent rests, etc). The physiotherapist will agree an individualised programme with the participant, indicate ways this could be progressed and provide guidance on keeping the daily play log and on setting/recording goals.

\section{Ongoing support and monitoring}

This will be followed by independent home use supported by a combination of telephone support (week 5-to identify and resolve any early difficulties encountered; week 12 to encourage progress, support people to overcome any barriers) and home review visits (weeks 7 and 16) at which the physiotherapist will reassess the individual and provide support and discuss possible progression/modification of the activities. Thereafter, ongoing monitoring, support and encouragement will be provided via monthly contact ( phone/email) from the physiotherapist.

modified as required. If patients experience a deterioration of their symptoms before the next review they can self-refer to the service. This may take the form of advice regarding relapse management or specific intervention.

The team operates a help-line between 9:00 and 11:00 Monday to Friday and there is an answer-phone out of hours. The team is available to discuss problems with patients and action appropriate treatments in a timely way. If therapy is required (occupational therapy/physiotherapy) this is offered by the MS specialist therapists and patients can be assessed and treated at home, as an outpatient, in work or a gym environment. Education, support and advice regarding disease modifying therapies, management of symptoms and carer support is available from the specialist nurse.

\section{Statistical analysis}

Recruitment and retention rates for the study and SDs of potential primary outcome measures will be 
estimated, and precision summarised using 95\% CIs. For each individual, adherence will be summarised ((the number of days on which activities were performed divided by the number of days recommended by the physiotherapist) $\times 100 \%$ ). These data will be collected via participant-completed daily play logs. We will estimate mean adherence $(\%)$ in the first 6 months of using the Wii by combining data from months $1-6$ for the immediate group and months 7-12 for the delayed group. We will estimate adherence in the second 6 months of using the Wii using data from months 7-12 in the immediate Wii group.

We will develop and test out data analysis procedures in preparation for developing a statistical analysis plan for a later RCT. This will involve (1) capitalising on the randomised nature of the study by comparing outcomes between study arms at 6 months (in months 1-6 the immediate Wii group will have been using the Wii, and the delayed group will not have been using the Wii), (2) analysing within person change over 6 months of using the Wii (ie, months 1-6 in the immediate group and months 7-12 in the delayed group) and (3) analysing within person change over 12 months of using the Wii (ie, months 1-12 in the immediate group).

\section{Qualitative analysis}

The interviews will be recorded, transcribed and thematically analysed using a generic qualitative approach ${ }^{126-131}$ and involving inter-researcher and service user interpretation/discussion. Free-text comments in the daily play logs will be collated and summarised. Themes developed will inform the design of a future trial and the selection of outcome measures.

\section{Participant withdrawal from study and/or from research follow-up}

If a participant withdraws from the study, the study researcher will be informed and will contact the participant. Provided the participant is willing to give a reason, the study researcher will find out why he/she wishes to withdraw from the study. The study researcher will also determine whether he/she wishes to continue to be included in the research follow-up or wishes to withdraw. If the participant wishes to withdraw from the research follow-up the study researcher will determine whether he/she gives permission to retain data collected before withdrawal for use at final analysis, or whether this information should be destroyed. No data will be used in the analysis without a participant's consent.

\section{Adverse events}

All AEs and their possible relation to the Mii-vitaliSe intervention will be closely monitored, documented and reported to the study Safety Monitoring Committee. The Safety Monitoring Committee will consider the events and offer advice to the project team. Participants will be asked to report all AEs related to use of the Wii to either the physiotherapists delivering Mii-vitaliSe or to the study researcher. These will be recorded on a case report form. In addition there will be a section on the daily play log to record AEs (including pain, tenderness, soreness, fatigue, dizziness, headaches, aching, stiffness, nausea, falls or near falls). The physiotherapists will ask participants whether they have experienced any AEs related to using the Wii in their monthly monitoring phone calls. The Dorset Multiple Sclerosis team will notify the research team of any AEs that become known to them.

The Chief Investigator will assess an AE to establish if it should be classified as a Serious Adverse Event (SAE) according to the National Research Ethics Service definition. If the $\mathrm{AE}$ is not defined as 'serious', it will be recorded in the study site file and the participant will be followed up by the research team. The AE will be documented in a participant's MS Service notes (where appropriate). Reports of related and unexpected SAEs will be submitted to the Research Ethics Committee within 15 days of the CI becoming aware of the event, using the 'Report of SAE form for non-CTIMPs' (V.3, April 2007), published on the NRES website. The sponsor will be notified within $24 \mathrm{~h}$.

\section{Project management and safety monitoring}

The study researcher will deal with the day-to-day management and coordination of the study and will call on other members of the project team as appropriate. The Chief Investigator will be responsible for the overall management of the project. The CI, study statistician, and study researcher will have regular meetings to discuss study progress. The study steering group will meet twice yearly to monitor progress. A Safety Monitoring Committee comprising two independent advisors (a neurologist with expertise in MS and clinical trials and a physiotherapist with expertise in risk assessment and management of service users with neurological disability) will review any AEs and make recommendations to the study steering group (eg, adapting the protocol/stopping the study, as necessary).

\section{Monitoring and audit}

The study will be monitored and audited in accordance with Poole Hospital NHS Foundation Trust (PHFT) policy. All trial related documents will be made available on request for monitoring and audit by PHFT and the Research Ethics Committee. Regular meetings will take place with the study monitor.

\section{Ethical considerations and dissemination}

The study has been reviewed and given a favourable opinion by the National Health Service (NHS) South Central Hampshire B Research Ethics Committee (ref: 12/SC/0420). Poole Hospital NHS Foundation Trust is acting as the sponsor. The study will be performed subject to Research Ethics Committee (REC) approval, including any provisions of Site Specific Assessment (SSA), and local Research and Development (R\&D) approval. This study will be conducted in accordance 
with the Research Governance Framework for Health and Social Care and GCP.

It is likely that majority of the participants in the study will have fatigue, and so we will try to minimise the impact that participation in the study has on their fatigue (eg, by staggering questionnaire load and providing a refreshment break during the physical assessments, offering taxi transport to the hospital).

We aim to disseminate the results of this research working closely with service user partners and the funder. It is anticipated that at least one publication in a peer reviewed journal will be produced and we intend to present the findings at a national and international conference. In collaboration with our service users we will produce a short report suitable for the general public. Further dissemination to pwMS will be via our unit's website, the Wiihabilitation website and (with their permission) MS charities and the Chartered Society of Physiotherapy. We also plan to disseminate findings via press releases and social media.

\section{DISCUSSION}

This pilot study will assess the acceptability and suitability of a home-based physiotherapist supported Wii intervention (Mii-vitaliSe) to increase activity levels in pwMS. Mii-vitaliSe addresses limitations highlighted in the literature by incorporating home-based activity, behavioural change principles, employing mixed methods and including a long term follow-up period. Findings from this study will establish whether a full scale RCT is feasible and, if supportive of proceeding to a full trial, will inform its design and conduct. The decision on whether to progress to a full trial will be made by the trial steering group. No specific criteria have been set. If found to be effective in a full trial, Mii-vitaliSe could increase activity levels in a group of people known to have low activity levels overall and could be readily implemented in a healthcare system such as the NHS. Technological developments in this field are ongoing with rehabilitation software currently being developed for the Nintendo Wii and other platforms. The general principles and findings from this research will have relevance for the next generation of software and platforms and also for other chronic conditions. ${ }^{71} 87$

\section{Author affiliations}

${ }^{1}$ Clinical Research Unit, School of Health and Social Care, Bournemouth University, Bournemouth, Dorset, UK

${ }^{2}$ School of Health and Social Care, Bournemouth University, Bournemouth, Dorset, UK

${ }^{3}$ Dorset Multiple Sclerosis Service, Poole Hospital NHS Foundation Trust, Poole, Dorset, UK

${ }^{4}$ Medical Physics Department, Poole Hospital NHS Foundation Trust, Poole, Dorset, UK

${ }^{5}$ Physiotherapy Department, National Star College, Cheltenham, Gloucestershire, UK

${ }^{6}$ Faculty of Health and Social Care, Hull University, Hull, Yorkshire, UK

Acknowledgements The authors would like to thank the individuals who attended the patient and public involvement (PPI) workshop to inform the development of the Mii-vitaliSe intervention. They also thank Mrs Jo Hickson and Mrs Kelly Saunders for attending the PPI workshop, for providing invaluable feedback about draft Mii-vitaliSe materials and the physical assessment protocol and for agreeing to be members of the study steering group. The authors also thank Dr Kate Jupp and Dr Mark Cossburn for agreeing to be members of the study Safety Monitoring Committee. They thank Dr Delva Shamley for commenting on an early version of the protocol.

Contributors ST, PT, CH, SC and SB were involved in the conception of the study and ST and PT led the design. ST with PT wrote the initial grant application and protocol. $\mathrm{CH}, \mathrm{SC}$ and SB advised on clinical aspects related to the grant application, SP led the medical physics component of the study, RS advised on technical aspects and KG advised on qualitative aspects. LF refined aspects of the draft protocol as part of her PhD studies. ST drafted the manuscript, LF and PT provided detailed feedback and all other authors critically reviewed and approved the final version.

Competing interests PT is a member of the MS Society Grant Review Panel for Care and Services Research. PT is also a member of the Advisory Board for the Sativex Registry. The Board provides an independent review of safety data for patients prescribed Sativex. Bournemouth University receives a fee from GW Pharma to cover time spent at meetings, and travel expenses.

Funding The study is funded by a project grant awarded by the Multiple Sclerosis Society in the UK (ref no. 933/10). The study is included in the National Institute of Health Research Clinical Research Network (NIHR CRN) portfolio (ID 13130).

Ethics approval NRES Committee South Central-Hampshire B (ref 12/SC/ 0420).

Provenance and peer review Not commissioned; peer reviewed for ethical and funding approval prior to submission.

Open Access This is an Open Access article distributed in accordance with the Creative Commons Attribution Non Commercial (CC BY-NC 4.0) license, which permits others to distribute, remix, adapt, build upon this work noncommercially, and license their derivative works on different terms, provided the original work is properly cited and the use is non-commercial. See: http:// creativecommons.org/licenses/by-nc/4.0/

\section{REFERENCES}

1. Rietberg MB, Brooks D, Uitdehaag BMJ, et al. Exercise therapy for multiple sclerosis. Cochrane Database Syst Rev 2004;(3): CD003980.

2. Motl RW, McAuley E, Snook EM. Physical activity and multiple sclerosis; a meta-analysis. Mult Scler 2005;11:459-63.

3. Dalgas U, Stenager E, Ingemann-Hansen T. Multiple sclerosis and physical exercise: recommendations for the application of resistance-, endurance- and combined training. Mult Scler 2008;14:35-53.

4. McConnell A. Can physical activity bring benefit to people with MS? Sport Exerc Scientist 2008;12:12-13.

5. Motl RW, Pilutti LA. The benefits of exercise training in MS. Nat Rev Neurol 2012;8:487-97.

6. Ponichtera-Mulcare JA. Exercise and multiple sclerosis. Med Sci Sport Exerc 1993;25:451-65.

7. Smith R, Adeney-Steel M, Fulcher GP, et al. Symptom change with exercise is a temporary phenomenon for people with multiple sclerosis. Mayo Clin Proc 2006;87:723-8.

8. Tallner A, Waschbisch A, Wenny I, et al. Multiple sclerosis relapses are not associated with exercise. Mult Scler 2012;18:232-5.

9. Tarakci E, Yeldan I, Huseyinsinoglu BE, et al. Group exercise training for balance, functional status, spasticity, fatigue and quality of life in multiple sclerosis: a randomized controlled trial. Clin Rehabil 2013;27:813-22.

10. Snook EM, Motl RW. Effect of exercise training on walking mobility in multiple sclerosis: a meta-analysis. Neurorehabil Neural Repair 2009;23:108-16.

11. Pilutti LA, Greenlee TA, Motl RW, et al. Effects of exercise training on fatigue in multiple sclerosis: a meta-analysis. Psychosom Med 2013;75:575-80.

12. Andreasen A, Stenager E, Dalgas U. The effect of exercise therapy on fatigue in multiple sclerosis. Mult Scler 2011;17:1041-54. 
13. Mostert S, Kesselring J. Effects of a short-term exercise training program on aerobic fitness, fatigue, health perception and activity level of subjects with multiple sclerosis. Mult Scler 2002;8:161-8.

14. Latimer-Cheung A, Pilutti LA, Hicks AL, et al. Effects of exercise training on fitness, mobility, fatigue, and health-related quality of life among adults with multiple sclerosis: a systematic review to inform guideline development. Arch Phys Med Rehabil 2013;94:1800-28.

15. Motl RW, Gosney JL. Effect of exercise training on quality of life in multiple sclerosis: a meta analysis. Mult Scler 2008;14:129-35.

16. Feinstein A, Rector N, Motl R. Exercising away the blues: can it help multiple sclerosis-related depression? Mult Scler 2013;14:1815-19.

17. Heesen C, Romberg A, Gold S, et al. Physical exercise in multiple sclerosis: supportive care or a putative disease-modifying treatment. Expert Rev Neurother 2006;6:347-55.

18. Dalgas U, Stenager E. Exercise and disease progression in multiple sclerosis: can exercise slow down the progression of multiple sclerosis? Ther Adv Neurol Disord 2012;5:81-95.

19. Marrie RA, Horwitz R, Cutter G, et al. High frequency of adverse health behaviors in multiple sclerosis. Mult Scler 2009;15:105-13.

20. Mulligan HF, Hale LA, Whitehead L, et al. Barriers to physical activity for people with long-term neurological conditions: a review study. Adapt Phys Activ Q 2012;29:243-65.

21. Becker H, Stuifbergen A. What makes it so hard? Barriers to health promotion experienced by people with multiple sclerosis and polio. Fam Community Health 2004;27:75-85.

22. Stroud N, Minahan C, Sabapathy S. The perceived benefits and barriers to exercise participation in persons with multiple sclerosis. Disabil Rehabil 2009;31:2216-22.

23. Borkoles E, Nicholls AR, Bell K, et al. The lived experiences of people diagnosed with multiple sclerosis in relation to exercise. Psychol Health 2008;23:427-41.

24. Vanner EA, Block $P$, Christodoulou CC, et al. Pilot study exploring quality of life and barriers to leisure-time physical activity in persons with moderate to severe multiple sclerosis. Disabl Health 2008;1:58-65

25. Asano M, Duquette $\mathrm{P}$, Andersen $\mathrm{R}$, et al. Exercise barriers and preferences among women and men with multiple sclerosis. Disabil Rehabil 2013:35:353-61.

26. Kayes NM. Physical activity engagement in people with multiple sclerosis $P h D$ [dissertation]. School of Rehabilitation and Occupation Studies, Auckland University of Technology, 2010.

27. Kayes NM, McPherson KM, Taylor D, et al. Facilitators and barriers to engagement in physical activity for people with multiple sclerosis: a qualitative investigation. Disabil Rehabil 2011;33:625-42.

28. Kayes NM, McPherson KM, Schluter PJ, et al. Exploring the facilitators and barriers to engagement in physical activity for people with multiple sclerosis. Disabil Rehabil 2011;33:1043-53.

29. McCullagh R, Fitzgerald AP, Murphy RP, et al. Long-term benefits of exercising on quality of life and fatigue in multiple sclerosis patients with mild disability: a pilot study. Clin Rehabil 2008;22:206-14

30. McAuley E, Motl RW, Morris KS, et al. Enhancing physical activity adherence and well-being in multiple sclerosis: a randomised controlled trial. Mult Scler 2007;13:652-9.

31. Plow MA, Resnik L, Allen SM. Exploring physical activity behaviour of persons with multiple sclerosis: a qualitative pilot study. Disabil Rehab 2009;31:1652-65.

32. Motl RW, Snook EM, McAuley E, et al. Correlates of physical activity among individuals with multiple sclerosis. Ann Behav Med 2006;32:154-61.

33. Smith C. Exercise friend or foe for people with multiple sclerosis who experience fatigue. N Z J Physiother 2012;40:29-31.

34. National Institute for Health and Care Excellence (NICE). Multiple Sclerosis: management of multiple sclerosis in primary and secondary care. Clinical Guideline 8.

35. Department of Health. The national service framework for long-term conditions. https://www.gov.uk/government/publications/ quality-standards-for-supporting-people-with-long-term-conditions (accessed 1 Feb 2014)

36. De Silva D. Helping people help themselves, a review of the evidence considering whether it is worthwhile to support self-management. London: The Health Foundation, 2011.

37. Sharma T. I own my MS, my MS doesn't own me. Synapse 2007;1:4-9.

38. Translating the NICE and NSF guidance into practice. A guide for physiotherapists. 2nd edn. MS Society, 2008:22.

39. Bandura A. A social-cognitive theory of personality. In: Pervin I, John $\mathrm{O}$, eds. Handbook of personality: theory and research. 2nd edn. New York: Guilford, 1999:154-96.
40. Bandura A. Self-efficacy: the exercise of self control. New York: Freeman, 1997.

41. Bandura A. Self-efficacy: toward a unifying theory of behavioura change. Psychol Rev 1977;84:191-215.

42. Motl RW, McAuley E, Doerksen S, et al. Preliminary evidence that self efficacy affects physical activity in multiple sclerosis. Int $J$ Rehabil Res 2009;32:260-3.

43. At least five a week. Evidence on the impact of physical activity and its relationship to health. A report from the Chief Medical Officer Department of Health, 2004. http://www.dh.gov.uk, http://www.dh. gov.uk

44. Motl RW, McAuley E, Snook EM, et al. Physical activity and quality of life in multiple sclerosis: intermediary roles of disability, fatigue, mood, pain, self efficacy and social support. Psychol Health Med 2009;14:111-24.

45. Betts L. Exercise and multiple sclerosis: have we come full circle? Br J Neurosci Nurs 2008;4:166-9.

46. Ferrier S, Dunlop N, Blanchard C. The role of outcome expectations and self-efficacy in explaining physical activity behaviours of individuals with multiple sclerosis. 2010 Behav Med 2010;36:7-11.

47. Riazi A, Thompson AJ, Hobart JC. Self-efficacy predicts self-reported health status in multiple sclerosis. Mult Scler 2004;10:61-6.

48. Ashford S, Edmunds J, French DP. What is the best way to change self-efficacy to promote lifestyle and recreational physical activity? A systematic review with meta-analysis. $\mathrm{Br} J$ Health Psychol 2010;15:265-88.

49. MS Trust: Open Door August 2008 Ann Minshull p. 14. A 63ro birthday surprise.

50. Baranowski T, Buday R, Thompson DI, et al. Playing for real: video games and stories for health-related behavior change. Am J Prev Med 2008;34:74-82.

51. Coyne C. Video "games" in the clinic: PTs report early results. PT Mag Phys Ther 2008;16:22-8.

52. Lyons EJ, Tate DF, Ward DS, et al. Engagement, enjoyment, and energy expenditure during active video game play. Health Psychol 2014;33:22-8.

53. Thornton M, Marshall S, McComas J, et al. Benefits of activity and virtual reality based balance exercise programmes for adults with traumatic brain injury: perceptions of participants and their caregivers. Brain Inj 2005;19:989-1000.

54. Vorhaus M. From gathering around the TV to gathering around the Wii. Advertising Age 2008;27:18.

55. Farrell L. Wii-habilitation. BMJ 2009;338:778.

56. Lanningham-Foster L, Fokster RC, McCrady SK, et al. Activity-promoting video games and increased energy expenditure. J Pediatr 2009;154:819-23.

57. Peng $\mathrm{W}$, Lin $\mathrm{J}-\mathrm{H}$, Crouse $\mathrm{J}$. Is playing exergames really exercising? a meta-analysis of energy expenditure in active video games. Cyberpsychol Behav Soc Netw 2011;14:681-8.

58. Griffin M, Shawis T, Impson R, et al. Comparing the energy expenditure of Wii $\mathrm{Fit}^{\mathrm{TM}}$-based therapy versus traditional physiotherapy games. Games Health J 2013;2:229-34.

59. Graves L, Stratton G, Ridgers ND, et al. Comparison of energy expenditure in adolescents when playing new generation and sedentary computer games: cross sectional study. BMJ 2007:335:1282-4.

60. Graves L, Stratton G, Ridgers ND, et al. Energy expenditure in adolescents playing new generation computer games. $\mathrm{Br} J$ Sports Med 2008;42:592-4.

61. Wiihabilitation [online]. http://wii-habilitation.blogspot.co.uk/2011/01/ does-rehab-kinect.htm (accessed 25 Jan 2014).

62. Warburton DER. The health benefits of active gaming: separating the myths from the virtual reality. Curr Cardiovasc Risk Rep 2013;7:251-5.

63. Nitz JC, Kuys S, Isles R, et al. Is the Wii Fit a new-generation too for improving balance, health and well-being? A pilot study. Climacteric 2010;13:487-91.

64. Wen CP, Wai JP, Tsai MK, et al. Minimum amount of physical activity for reduced mortality and extended life expectancy: a prospective cohort study. Lancet 2011;378:1244-53.

65. Motl RW, Arnett PA, Smith MM, et al. Worsening of symptoms is associated with lower physical activity levels in individuals with multiple sclerosis. Mult Scler 2008;14:140-2.

66. Kerrigan DJ, Chen $\mathrm{H}$, Wiederhold MD, et al. Evaluation of the Nintendo Wii for physical exercise and rehabilitation. Cyberpsychol Behav 2009;12:107.

67. Sparks D, Chase D, Coughlin L. Wii have a problem a review of self-reported Wii related injuries. Inform Prim Care 2009;17:55-7. 
68. Taylor MJD, McCormick D, Shawis T, et al. Activity-promoting gaming systems in exercise and rehabilitation. $J$ Rehabil Res Dev 2011;48:1171-86.

69. Nintendo. Wii operations manual. Redmond, Washington: Nintendo of America, 2006.

70. Plow M, Finlayson M. A qualitative study exploring the usability of Nintendo Wii Fit among persons with multiple sclerosis. Occup Ther Int 2014;21:21-32.

71. Pessoa TM, Coutinho DS, Pereira VM, et al. The Nintendo Wii as a tool for neurocognitive rehabilitation, training and health promotion. Comput Hum Behav 2014;31:384-92.

72. Deutsch JE, Borbely M, Filler J, et al. Use of a low-cost commercially available gaming console (Wii) for rehabilitation of an adolescent with cerebral palsy. Phys Ther 2008;88:1196-207.

73. Laver K, George S, Ratcliffe J, et al. Use of an interactive video gaming program compared with conventional physiotherapy for hospitalised older adults: a feasibility trial. Disabil Rehabil 2012;34:1802-8

74. Laver KE, George S, Thomas S, et al. Virtual reality for stroke rehabilitation. Cochrane Database Syst Rev 2011;(9):CD008349.

75. Esculier JF, Vaudrin J, Bériault $P$, et al. Home-based balance training programme using Wii Fit with balance board for Parkinson's disease: a pilot study. J Rehabil Med 2012;44:144-50.

76. Saposnik G, Mamdani M, Bayley M, et al. Effectiveness of Virtual Reality Exercises in Stroke Rehabilitation (EVREST): rationale, design, protocol and baseline data of a pilot randomized clinical trial assessing the Wii gaming system. Int J Stroke 2010;5:47-51.

77. Saposnik G, Teasell R, Mamdani M, et al. Effectiveness of virtual reality using Wii gaming technology in stroke rehabilitation: a pilot randomized clinical trial and proof of principle. Stroke 2010;41:1477-84

78. Pompeu JE, Mendes FA, Silva KG, et al. Effect of Nintendo Wii ${ }^{\text {TM}}$-based motor and cognitive training on activities of daily living in patients with Parkinson's disease: a randomised clinical trial. Physiotherapy 2012;98:196-204.

79. Fung V, Ho A, Shaffer J, et al. Use of Nintendo Wii Fit' ${ }^{\mathrm{TM}}$ in the rehabilitation of outpatients following total knee replacement: a preliminary randomised controlled trial. Physiotherapy 2012;98:183-8.

80. Gil-Gómez J-A, Lloréns R, Alcañiz M, et al. Effectiveness of a Wii balance board-based system (eBaViR) for balance rehabilitation: pilot randomized clinical trial in patients with acquired brain injury. $J$ Neuroeng Rehabil 2011;8:30.

81. Yuen HK, Holthaus K, Kamen DL, et al. Using Wii Fit to reduce fatigue among African American women with systemic lupus erythematosus: a pilot study. Lupus 2011;20:1293-9.

82. Kempf K, Martin S. Autonomous exercise game use improves metabolic control and quality of life in type 2 diabetes patients-a randomized controlled trial. BMC Endocrinol Disord 2013;13:57.

83. Brichetto G, Spallarossa P, de Carvalho ML, et al. The effect of Nintendo® Wiiß on balance in people with multiple sclerosis: a pilot randomized control study. Mult Scler 2013;19:1219-21.

84. Prosperini L, Fortuna D, Giannì C, et al. Home-based balance training using the Wii balance board: a randomized, crossover pilot study in multiple sclerosis. Neurorehabil Neural Repair 2013;27:516-25.

85. Nilsagård YE, Forsberg AS, von Koch L. Balance exercise for persons with multiple sclerosis using Wii games: a randomised controlled multi-centre study. Mult Scler 2013;19:209-16.

86. Plow M, Finlayson M. Potential benefits of Nintendo Wii Fit among people with multiple sclerosis. Int J MS Care 2011; 13:21-30.

87. Plow MA, McDaniel C, Linder S, et al. Scoping review of exergaming for adults with systemic disabling conditions. J Bioeng Biomed Sci 2011;S1:002.

88. Hale LA, Smith $\mathrm{C}$, Mulligan $\mathrm{H}$, et al. "Tell me what you want, what you really really want....": asking people with multiple sclerosis about enhancing their participation in physical activity. Disabil Rehabil 2012;34:1887-93.

89. Craig P, Dieppe P, Macintyre S, et al. Developing and evaluating complex interventions: the new Medical Research Council guidance. BMJ 2008;337:a1655. http://www.mrc.ac.uk/ complexinterventionsguidance. MRC guidelines.

90. Leon AC, Davis LL, Kraemer HC. The role and interpretation of pilot studies in clinical research. J Psychiatr Res 2011;45:626-9.

91. INVOLVE. Briefing notes for researchers: involving the public in NHS, public health and social care research. Eastleigh: INVOLVE, 2012.

92. Lancaster GA, Dodd S, Williamson PR. Design and analysis of pilot studies: recommendations for good practice. J Eval in Clin Pract 2004;10:307-12.
93. Thomas $\mathrm{S}$, Thomas $\mathrm{PW}$, Kersten $\mathrm{P}$, et al. A pragmatic parallel arm multi-centre randomised controlled trial to assess the effectiveness and cost-effectiveness of a group-based fatigue management programme (FACETS) for people with multiple sclerosis. J Neurol Neurosurg Psychiatry 2013;10:1092-9.

94. Browne $\mathrm{RH}$. On the use of a pilot sample for sample size determination. Stat Med 1995;14:1933-40.

95. Orme M, Kerrigan J, Tyas D, et al. The effect of disease, disability and relapses on the utility of people with multiple sclerosis in the UK. Value Health 2007;10:54-60.

96. Kurtzke JF. Rating neurologic impairment in multiple sclerosis: an expanded disability status scale (EDSS). Neurology 1983;33:1444-52.

97. Stokes M, Stack E, eds. Physical management for neurological conditions. 3rd edn. Edinburgh: Churchill Livingston, 2011.

98. Morris ME, Cantwell C, Vowels $L$, et al. Changes in gait and fatigue from morning to afternoon in people with multiple sclerosis. J Neurol Neurosurg Psychiatry 2002;72:361-5.

99. Rossier P, Wade DT. Validity and reliability comparison of 4 mobility measures in patients presenting with neurologic impairment. Arch Phys Med Rehabil 2001;82:9-13.

100. Hill KD, Bernhardt J, McGann AM, et al. A new test of dynamic standing balance for stroke patients: reliability, validity and comparison with healthy elderly. Physiother Can 1996;48: 257-62.

101. Frzoviv D, Morris ME, Vowels L. Clinical tests of standing balance; performance of persons with multiple sclerosis. Arch Phys Med 2000;81:215-21.

102. Cattaneo D, Regola A, Meotti M. Validity of six balance disorders scales in persons with multiple sclerosis. Disabil Rehabil 2006;28:789-95.

103. Perring S, Summers T. Laboratory-free measurement of gait rhythmicity in the assessment of the degree of impairment and the effectiveness of rehabilitation in patients with vertigo resulting from vestibular hypofunction. Physiol Meas 2007;28:697-705.

104. Perring S, Jepson R. The use of a two-dimensional ultrasound time-of-flight system for location of the centre of gravity in posturography measurements. Physiol Meas 2001;22:447-52.

105. Godin G, Shephard RJ. A simple method to assess exercise behaviour in the community. Can J Appl Sport Sci 1985; 10:141-6.

106. Gosney JL, Scott JA, Snook EM, et al. Physical activity and multiple sclerosis. Validity of self-report and objective measures. Fam Comm Health 2007;30:144-50.

107. Ryan CG, Grant PM, Tigbe WW, et al. The validity and reliability of a novel activity monitor as a measure of walking. Br J Sports Med 2006;40:779-7844.

108. Mathiowetz V, Weber K, Kashman N, et al. Adult norms for the nine-hole peg test of finger dexterity. Occup Ther $J$ Res 1985;5:24-37.

109. Kroll T, Kehn M, Ho PS, et al. The SCI Exercise Self-Efficacy Scale (ESES): development and psychometric properties. Int J Behav Nutr Phys Act 2007;4:34.

110. Schwartz CE, Coulthard-Morris L, Zeng Q, et al. Measuring self-efficacy in people with multiple sclerosis: A validation study. Arch Phys Med Rehabil 1996;77:394-8.

111. Zigmond AS, Snaith RP. The Hospital Anxiety and Depression Scale. Acta Psychiatr Scand 1983;67:361-70.

112. The EuroQol Group. EuroQol-a new facility for the measurement of health-related quality of life. Health Policy 1990; 16:199-208.

113. Hobart J, Lamping D, Riazi A. The multiple sclerosis impact scale (MSIS-29): a new patient-based outcome measure. Brain 2001;124:962-73.

114. Hobart JC, Riazi A, Lamping D, et al. Improving the evaluation of therapeutic interventions in multiple sclerosis: development of a patient-based measure of outcome. Health Technol Assess 2004:8:9.

115. Hann DM, Jacobsen PB, Azzarello LM, et al. Measurement of fatigue in cancer patients: development and validation of the Fatigue Symptom Inventory. Qual Life Res 1998;74:301-10.

116. Ware JE, Sherbourne CD. The MOS 36-item Short-Form health survey (SF-36):I. Conceptual framework and item selection. Med Care 1992;30:473-83.

117. Mihalko SL, Brenes GA, Farmer DF, et al. Challenges and innovations in enhancing adherence. Control Clin Trials 2004;25:447-57

118. Martin KA, Bowen DJ, Dunbar-Jacob J, et al. Who will adhere? Key issues in the study and prediction of adherence in randomized controlled trials. Control Clin Trials 2000;21:195S-9S. 
119. Norton K, Norton L, Sadgrove D. Position statement on physical activity and exercise intensity terminology. J Sci Med Sport 2010;13:496-502.

120. Borg GA. Psychophysical bases of perceived exertion. Med Sci Sports Exerc 1982;14:377-81.

121. Materials from the Wiihabilitation website. http://www.wiihabilitation co.uk/ (accessed 1 Feb 2014).

122. Deutsch JE, Brettler A, Smith C, et al. Nintendo Wii sports and Wii Fit game analysis, validation, and application to stroke rehabilitation. Top Stroke Rehabil 2011;18:701-19.

123. Crooks N. Guidelines for using the Wii with patients in the West Midlands Rehabilitation Centre. Produced by South Birmingham Primary Care Trust and circulated via the Chartered Physiotherapist website. 2010. http://www.csp.org.uk/

124. Advisory document (created by Hirons C [Clinical Specialist Physiotherapist], Smith O [Clinical Specialist Prosthetics], Hirons R [Clinical Specialist Prosthetics] on the suitability of Wii ${ }^{\mathrm{TM}}$ Fit exercises for lower limb prosthetic users either as a recreation or rehabilitation tool. 2009. http://www.wiihabilitation.co.uk/files/wii_fit_ bro_uk_traffic_light_version.pdf
125. 'Wii play it safe' document created by an occupational therapist. Hinkel, MW. http://physical-therapy.advanceweb.com/Article/ Wii-Play-it-Safe-2.aspx

126. Strauss A, Corbin J. Basics of qualitative research; techniques and procedures for developing grounded theory. 2nd edn. Thousand Oaks, CA: Sage, 1998.

127. Galvin K. Navigating a qualitative course in programme evaluation. In: Holloway I, ed. Qualitative research in health care. Maidenhead: Open University Press, 2005:229-49.

128. Caelii K, Rya L, Mill J. 'Clear as mud' toward greater clarity in generic qualitative research. Int J Qual Methods 2003;2: $1-13$.

129. Kahlke RM. Pitfalls and benefits of methodology mixology. Int $J$ Qual Methods 2014;13:37-52.

130. Mason J. Qualitative interviewing: asking, listening and interpreting. In: May T, ed. Qualitative research in action. London: Sage, 2002:225-41.

131. Bowling A. Research methods in health: investigating health and health services. 2nd edn. Buckingham: Open University Press, 2002 